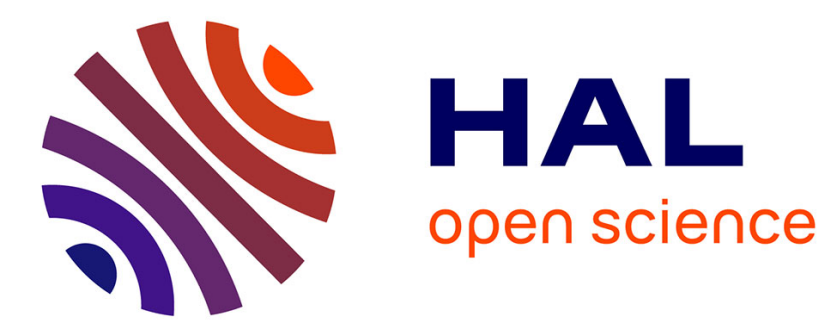

\title{
Conformations of polydisperse polymer solutions : bimodal distribution
}

\author{
J.-F. Joanny, P. Grant, L.A. Turkevich, P. Pincus
}

\section{To cite this version:}

J.-F. Joanny, P. Grant, L.A. Turkevich, P. Pincus. Conformations of polydisperse polymer solutions: bimodal distribution. Journal de Physique, 1981, 42 (7), pp.1045-1051. 10.1051/jphys:019810042070104500 . jpa-00209081

\section{HAL Id: jpa-00209081 https://hal.science/jpa-00209081}

Submitted on 1 Jan 1981

HAL is a multi-disciplinary open access archive for the deposit and dissemination of scientific research documents, whether they are published or not. The documents may come from teaching and research institutions in France or abroad, or from public or private research centers.
L'archive ouverte pluridisciplinaire HAL, est destinée au dépôt et à la diffusion de documents scientifiques de niveau recherche, publiés ou non, émanant des établissements d'enseignement et de recherche français ou étrangers, des laboratoires publics ou privés. 
Classification

Physics Abstracts

$36.20 \mathrm{C}-61.40 \mathrm{~K}$

\title{
Conformations of polydisperse polymer solutions : bimodal distribution
}

\author{
J. F. Joanny \\ Physique de la Matière Condensée, Collège de France, 75231 Paris Cedex 05, France \\ P. Grant, L. A. Turkevich \\ Department of Physics, University of California, Los Angeles, California 90024, U.S.A.
}

and P. Pincus

Department of Physics, University of California, Los Angeles, California 90024, U.S.A. and Ecole Supérieure de Physique et de Chimie Industrielles, 75231 Paris Cedex 05, France

(Reçu le 8 septembre 1980, révisé le 16 mars 1981, accepté le 17 mars 1981)

\begin{abstract}
Résumé. - Partant de la théorie de Flory pour une chaîne linéaire flexible de $N$ monomères dans un polymère fondu de chaînes plus courtes ( $P$ monomères) on emploie des arguments d'échelle pour établir le « diagramme de phase " d'une solution bimodale athermique (système ternaire composé de chaînes $N$, de chaînes $P$ et d'un solvant). Qualitativement la présence des chaînes courtes peut s'interpréter comme une réduction de la qualité du solvant effectif qui entoure les longues chaînes. On indique des lois asymptotiques pour le rayon de giration dans les différentes régions de ce diagramme de phase.
\end{abstract}

\begin{abstract}
Starting with the Flory theory for one long flexible polymer chain of $N$ segments in a homopolymer melt of shorter ( $P$ segments) chains, we employ scaling arguments to construct the " phase diagram " of an athermal bimodal solution, i.e. a ternary system composed of $N$ chains, $P$ chains, and solvent. Qualitatively the presence of short chains reduces the effective solvent quality as experienced by the long chains. We present limiting forms for the radii of gyration of both chains through out the " phase diagram ".
\end{abstract}

1. Introduction. - Recent scaling theories [1] of linear polymer systems in solution examine the idealized case where all the chains have identical molecular weight. Physical systems, however are invariably more or less polydisperse. In order to determine how serious the consequences of polydispersity may be, we have studied the case of a bimodal distribution of polymer chains. We utilize scaling concepts to predict the conformation of polymers for a bimodal distribution of linear polymers in an athermal solvent. These results then may be extended to give a qualitative prediction for the behaviour of more general polydisperse systems.

In the second section, we review the well established results [2] of the conformation of polymers in a melt. A single long polymer (degree of polymerization $N$ ) in a melt of sufficiently short polymers $(D P=P)$ is expanded relative to a random walk chain, while in a homogeneous melt, the polymers are not expand-

LE JOURNAL DE PHYSIQUE - T. 42, No 7, JUILLET 1981 ed. The Flory condition for swelling of the long chain is

$$
P<N^{1 / 2} .
$$

Starting with the isolated long chain radius, we derive the concentration dependence of the $N$ chain swelling. The short chains are never swollen in the melt.

In the third section, we generalize these results to a ternary system of $N$ chains, $P$ chains, and athermal solvent.

2. Bimodal melt. - Before we investigate the bimodal distribution of polymers in a solvent, we review the simpler case of a melt. The polymers are assumed to be chemically identical, differing only in the degree of polymerization, the long polymers having $N$ monomeric units, the short polymers having $P$ monomeric units.

For convenience, we place the chains on a threedimensional Flory-Huggins Lattice with lattice para- 
meter $a$. Each polymer is described by a random walk along adjacent sites subject to the exclusion of occupied sites; each site of the lattice is occupied by one and only one monomeric unit. The fraction of sites occupied by monomeric units belonging to long and short chains are respectively the volume fractions $\phi_{N}$ and $\phi_{P}$. These volume fractions are related to the densities $\rho_{N}$ and $\rho_{P}$ of monomeric units (the number of monomeric units per unit volume) by $\phi_{N}=a^{3} \rho_{N}$ and $\phi_{P}=a^{3} \rho_{P}$. In a melt, all sites are occupied and hence

$$
\phi_{N}+\phi_{P}=1 \text {. }
$$

The short polymers always execute a random walk $R_{P} \sim P^{1 / 2} a$. We thus confine our investigation to the conformation of the long polymers.

a) ONE LONG POLYMER IN A SHORT POLYMER MELT. - First we calculate the conformation of one long polymer in a melt of short polymers $\left(\phi_{P} \sim 1\right)$.

The free energy associated with the long chain has two contributions : the elastic energy of the long chain, which favours the random walk configuration, and the entropy of mixing, which favours a swelling of the long chain. Assuming a Gaussian distribution of monomeric units, the elastic free energy of a chain of radius $R$ is given by $\left(^{1}\right)$

$$
F=\frac{3 R^{2}}{2 R_{0}^{2}} T-3 T \ln \frac{R}{R_{0}}
$$

where $R_{0}^{2}=N a^{2}$ is the mean square radius for the random walk.

The translational entropy per site associated with the possible configurations of polymers on the lattice is given by

$$
-\left.S\left(\phi_{N}\right)\right|_{\text {site }}=\frac{\phi_{N}}{N} \ln \frac{\phi_{N}}{N}+\frac{\left(1-\phi_{N}\right)}{P} \ln \frac{\left(1-\phi_{N}\right)}{P}
$$

where the two terms represent the translational entropy of the centre of mass of long and short chains respectively. The entropy of mixing, $S_{\text {mix }}$, is defined as the full translational entropy minus the weighted average of the entropies of pure long and pure short chains

$$
\left.S_{\text {mix }}\right|_{\text {site }}=\left.S\left(\phi_{N}\right)\right|_{\text {site }}-\left.\phi_{N} S(1)\right|_{\text {site }}-\left.\phi_{P} S(0)\right|_{\text {site }} .
$$

Substitution from (4) yields $\left({ }^{2}\right)$

$$
-\left.S_{\text {mix }}\left(\phi_{N}\right)\right|_{\text {site }}=\frac{1-\phi_{N}}{P} \ln \left(1-\phi_{N}\right) .
$$

( $\left.{ }^{1}\right)$ We use units such that the Boltzmann constant $k_{\mathrm{B}}=1$.

(2) In (6), we neglect the term $\phi_{N} / N \ln \phi_{N}$ which represents the translational entropy of a single $N$ chain and has no effect on the polymer radius in the thermodynamic limit.
Combining the elastic energy (3) with the entropy of mixing (6), where the total number of sites is $4 \pi R^{3} / 3 a^{3}$, we obtain the free energy of the long chain

$$
\frac{\dot{F}}{T}=\frac{3 R^{2}}{2 N a^{2}}-3 \ln \frac{R}{R_{0}}+\frac{R^{3}}{a^{3}}\left[\frac{1-\phi_{N}}{P} \ln \left(1-\phi_{N}\right)\right]
$$

where we omit some numerical factors [3]. Since there is only a single long chain, $\phi_{N}=N a^{3} / R^{3}$ is small, and we may expand (7) to obtain

$\frac{F}{T}=\frac{3}{2} \frac{R^{2}}{N a^{2}}+P^{-1}\left(\frac{R}{a}\right)^{3} \phi_{N}^{2}+3 \ln (a / R)-$ constant.

Minimizing this free energy $(\partial(F / T) / \partial R=0)$ to determine the radius of the long polymer, we obtain

$$
\frac{R}{N a^{2}}-\frac{N^{2} a^{3}}{P R^{4}}-\frac{1}{R}=0 \text {. }
$$

Relation (9) may alternatively be derived by equating the chemical potentials of the short chains inside and outside the sphere of the long chain [4]

$$
\left(\mu_{P}^{\text {in }}=-\partial(F / V) / \partial \phi_{P}^{\text {in }} \quad \text { and } \quad \mu_{P}^{\text {out }}=-\partial(F / V) / \partial \phi_{P}^{\text {out }}\right) .
$$

For $P<N^{1 / 2}$, we neglect the third term in (9) and obtain

$$
R=N^{3 / 5} P^{-1 / 5} a
$$

Thus the long chain is expanded relative to a random walk chain $\left(R / R_{0}>1\right)$, as first predicted by Flory [2]. This situation may be interpreted physically as a single long polymer immersed in a solvent of short polymers, i.e. the short chains behave as a reasonably good solvent for the long chains. However, compared to the expansion in an athermal solvent $\left(R=N^{3 / 5} a\right)$, the short polymer solvent is weaker by a factor of $P^{-1 / 5}$. This expansion of the long chain has been qualitatively verified by neutron scattering of protonated long chains in a melt of deuterated short chains [5]. The result, equation (10), may be understood on terms of a chain of renormalized [6] hard beads of dimension $\lambda$

$$
\lambda=P a \text {. }
$$

The distance scale $\lambda$ is determined as the chain radius where the Flory radius (10) is equal to the ideal radius $R_{0}$.

For distances $r<\lambda$ the chain is ideal, i.e. while for $r>\lambda$ the correlations are that of an excluded volume chain. Note that for an athermal solvent $(P \rightarrow 1), \lambda \rightarrow a$ and the chain is swollen on all length scales. There exist $g_{\lambda}=P^{2}$ monomers per bead which leads to a chain radius

$$
R=\left(N / g_{\lambda}\right)^{3 / 5} \lambda
$$


which is identical to equation (10). The $N$ chain in the $P$ melt may be considered as an excluded volume chain in an athermal solvent having $N_{\lambda}=N / g_{\lambda}$ beads each of radius $\lambda$.

For $P>N^{1 / 2}$, the second term in (13) may be neglected, yielding

$$
R=N^{1 / 2} a .
$$

This is just the result for a homogeneous polymer melt. Physically, the short chains may extend through the sphere occupied by the long chain and the distinction of lying with the $N$ sphere or outside of it becomes fuzzy. The melt thus looks homogeneous, and there is no expansion of the long chain. Note that equations (10) and (12) crossover properly to equation (13) as $P \rightarrow N^{1 / 2}$.

b) Semi-dilute $N$ chains in a $P$ melt. - We now consider a bimodal melt containing a finite density $\phi_{N}$ of long polymers $\left(N>P^{2}\right)$.

It is convenient to use the volume fraction $\tilde{\phi}_{N}$ occupied by the renormalized beads

$$
\tilde{\phi}_{N}=\left(\phi_{N} / g_{\lambda}\right)(\lambda / a)^{3}=\phi_{N} P .
$$

The $N$ chains begin to overlap at a critical bead concentration $\widetilde{\phi}_{N}^{*}$ given by

$$
\tilde{\phi}_{N}^{*}=N_{\lambda}\left(\lambda / R_{N}\right)^{3}=N_{\lambda}^{-4 / 5} .
$$

In terms of the renormalized variables $\left(\tilde{\phi}_{N}, N_{\lambda}, \lambda\right)$ the semi-dilute bimodal melt maps into a semi-dilute athermal polymer solutions. For $\tilde{\phi}_{N}>\tilde{\phi}_{N}^{*}$, the $N$ chains screen out the swelling on large scales; i.e. there exists a correlation length [7, 8] $\xi_{N}$ such that for $r<\xi_{N}$ the correlations are those of a swollen chain but units of size $\xi_{N}$ obey random walk statistics. We determine the blob size $\xi_{N}$ and the number of beads per blob $g_{N}$ by

$$
\begin{aligned}
\tilde{\phi}_{N} & =g_{N}\left(\lambda / \xi_{N}\right)^{3} \\
\xi_{N} & =g_{N}^{3 / 5} \lambda .
\end{aligned}
$$

The condition (16) may be interpreted as follows. The force tending to swell the $N$ chain is associated with the two body repulsions between beads (8) and is therefore proportional to $-\frac{\mathrm{d} \rho(r)}{\mathrm{d} r}$ where $\rho(r)$ is the density of beads at a distance $r$ from the centre of gravity of the $N$ chain. For large $r, \rho(r)$ tends toward the average bead concentration $\widetilde{\phi}_{N} \lambda^{-3}$. For an isolated chain

$$
\rho_{0}(r)=n / r^{3} \quad r<R_{N}
$$

where $n$ is the number of beads in a sphere of radius $r$. From figure 1 , where $\rho(r)$ and $\rho_{0}(r)$ are sketched, we note that the swelling force $\left(\sim-\frac{\partial \rho}{\partial r}\right)$ tends toward zero in the vicinity of

$$
\rho_{0}\left(\xi_{N}\right)=\tilde{\phi}_{N} \lambda^{-3}
$$

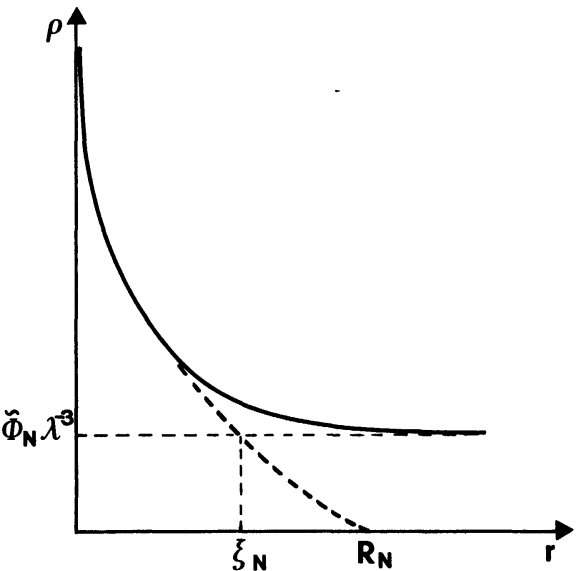

Fig. 1. - A sketch of the $N$ chain bead density $\rho(r)$ in a semi-dilute bimodal melt, at a distance from the centre of gravity of an $N$ chain. The dashed curve $\rho(r)$ represents the density for an isolated $N$ chain.

which is (16). Equation (17) reflects the requirement that for $r<\xi_{N}$, the chain is swollen. Combining (16) and (17), we obtain

$$
\xi_{N}=\tilde{\phi}_{N}^{-3 / 4} \lambda=\phi_{N}^{-3 / 4} P^{3 / 4} a .
$$

Note that at the critical overlap concentration $\tilde{\phi}_{N}^{*}$,

$$
\xi_{N}=R_{N} \text {. }
$$

For $\tilde{\phi}_{N}>\tilde{\phi}_{N}^{*}$, the chain radius is that of a random walk chain of blobs

$$
\begin{aligned}
R_{N} & =\left(N_{\lambda} / g_{N}\right)^{1 / 2} \xi_{N}=N_{\lambda}^{1 / 2} \tilde{\phi}_{N}^{-1 / 8} \lambda \\
& =N^{1 / 2}\left(P \phi_{N}\right)^{-1 / 8} a .
\end{aligned}
$$

As the concentration $\phi_{N}$ increases further, the chain becomes ideal on all scales where $\xi_{N}=\lambda$ which is equivalent to $\tilde{\phi}_{N}=P \phi_{N}=1$. These results are summarized in table I.

Table I. - The conformation of long polymers in a bimodal melt, $P<N^{1 / 2}$.

\begin{tabular}{|l|l|}
\hline \multicolumn{1}{|c|}{$\begin{array}{c}\text { Concentration } \\
\text { of long chains }\end{array}$} & Radius of long chains \\
\hline$N^{-4 / 5} P^{3 / 5}>\phi_{N}$ & $R_{N}=N^{3 / 5} P^{-1 / 5} a$ \\
\hline$N^{-4 / 5} P^{3 / 5}<\phi_{N}<P^{-1}$ & $R=N^{1 / 2} P^{-1 / 8} \phi_{N}^{-1 / 8} a$ \\
\hline$P^{-1}<\phi_{N}$ & $R=N^{1 / 2} a$ \\
\hline
\end{tabular}

3. Bimodal solution. - In this section, we generalize the results of the preceding section for the bimodal melt to the case of a bimodal solution; i.e. a ternary system of $N$ chains, $P$ chains, and athermal solvent. In fact, we shall generate the various regions in the $\left(\phi_{N}, \phi_{P}\right)$ plane having differing conformational beha- 
viours with regard to the $P$ and $N$ chain radii. It will prove convenient to measure $\phi_{N}$ and $\phi_{P}$ relative to the individual $N$ and $P$ chain overlap concentrations $\phi_{N}^{*}$ and $\phi_{P}^{*}$ in an athermal solvent where

$$
\phi_{x}^{*}=N_{x}^{-4 / 5} ; \quad \alpha=N, P
$$

i.e. we utilize reduced densities

$$
\Phi_{\alpha}=\phi_{x} / \phi_{x}^{*} ; \quad \alpha=N, P .
$$

Our strategy is to first treat the cases of dilute $N$ chains $\left(\Phi_{N}<1\right)$ as a function of $\Phi_{P}$; next dilute $P$ chains as a function of $\Phi_{N}$; then to use scaling arguments to construct proper crossovers in the remainder of the $\left(\Phi_{N}, \Phi_{P}\right)$ plane.

a) Dilute $N$ Chains. - We now investigate the swelling of one long $N$ chain immersed in an athermal solution of shorter $P$ chains. This problem has already been studied by De Gennes [9] by slightly different arguments. Following De Gennes we will distinguish three regimes for the chain concentration $\Phi_{p}$.

i) a regime $\Phi_{P}<1$ where the $P$ chains are dilute and the $N$ chain is swollen;

ii) a regime $1<\Phi_{P}<\Phi_{P}^{+}$(with $\Phi^{+}=(N / P)^{4 / 5}$ ) where the $P$ chains are semi-dilute but the $N$ chain is still swollen;

iii) a regime $\Phi_{P}>\Phi^{+}$where the $P$ chains are semi-dilute and the $N$ chain is ideal.

\section{i) Dilute solution of $P$ chains}

In this limit, F. Brochard [10] has shown that there is no segregation of the $P$ chain outside and inside the long $N$ chain. The concentration of $P$ chains is uniform. The radius of the $P$ chain is the Flory radius for a chain in an athermal solvent $R_{P}=P^{3 / 5} a$. A generalization of Flory's mean field theory [9] to the case of a mixed solvent yields an effective medium dimensionless interaction parameter $u=\left(1+P \phi_{P}\right)^{-1}$. This is derived by a different method in the appendix. In order to study the swelling of the long chain, it is convenient to renormalize the unit length $a$ to $R_{P}$ i.e. to view the $N$ chain of blobs of size $R_{P}$.

At length scales smaller than $R_{P}$ the $N$ chain behaves exactly the same way as the $P$ chains do and shows excluded volume effects.

At length scales larger than $R_{P}$ we are left in our renormalized units with a ternary system of one $N$ chain of $N / P$ units in a solvent of the same chemical species occupying a volume fraction $\phi=\frac{\rho_{P}}{P} R_{P}^{3}=\Phi_{P}$ $(\ll 1)$ and an athermal solvent. The $N$ chain is thus swollen and shows a dimensionless excluded volume parameter $u=\frac{1}{1+\Phi_{P}}$.

Its radius is then

$$
R_{N}=\left(\frac{N}{P}\right)^{3 / 5} R_{P}\left(1-\Phi_{P}\right)^{1 / 5}=N^{3 / 5} a\left(1-\Phi_{P}\right)^{3 / 5} .
$$

\section{ii) Semi-dilute $P$ solution}

We turn now to the case of a semi-dilute $P$ chain solution where the correlation length $\xi_{P}=\left(c_{P} a^{3}\right)^{-3 / 4} a$.

It is here natural to renormalize the unit length to $\xi_{P}$, i.e. to introduce blobs of $g_{P}$ monomers such that $\xi_{P}=g_{P}^{3 / 5} a$. We need to estimate the dimensionless excluded volume parameter $u$ between blobs; it is here proportional to $u=\frac{1}{1+P_{\mathrm{b}} \Phi_{\mathrm{b}}}$ where $P_{\mathrm{b}}$ is the number of blobs in a $P$ chain and $\Phi_{\mathrm{b}}$ the volume fraction occupied by the blobs

$$
u=\frac{g_{P}}{P} .
$$

The Flory free energy for the chain of $N / g_{P}$ blobs is then

$$
\frac{F}{T}=\frac{R_{N}^{2}}{\frac{N}{g_{P}} \xi_{P}^{2}}+u \xi_{P}^{3} \frac{\left(N / g_{P}\right)^{2}}{R_{N}^{3}} .
$$

The chain is swollen if

$$
u \xi_{P}^{3}\left(\frac{N}{g_{P}}\right)^{2} \frac{1}{R_{0 N}^{3}} \gg 1: R_{0 N}^{2}=\frac{N}{g_{P}} \xi_{P}^{2}
$$

which recovers De Gennes result

$$
\Phi_{P}<\Phi^{+}=\left(\frac{N}{P}\right)^{4 / 5}
$$

The radius of the chain is given by the Flory formula

$$
R_{N}=\left(\frac{N}{g_{P}}\right)^{3 / 5} \xi_{P} u^{1 / 5}
$$

iii) The interactions are screened if

$$
\frac{u \xi_{P}^{3}\left(\frac{N}{g_{P}}\right)^{3}}{R_{0 N}^{3}} \ll 1
$$

(i.e. $\Phi_{P} \gg \Phi^{+}$) and the radius is

$$
R_{N}=\left(\frac{N}{g_{P}}\right)^{1 / 2} \xi_{P} .
$$

These results for $\Phi_{N}<1$ are summarized in table II.

Table II. - Conformations for dilute $N$ chains, $\Phi_{N}<1$.

\begin{tabular}{|l|l|l|}
\hline \multicolumn{1}{|c|}{$\Phi_{P}$} & $R_{P} / R_{\mathrm{F}}^{P}$ & \multicolumn{1}{|c|}{$R_{N} / R_{\mathrm{F}}^{N}$} \\
\hline$\Phi_{P}<1$ & 1 & 1 \\
\hline$\Phi^{+}>\Phi_{P}>1$ & $\Phi_{P}^{-1 / 8}$ & $\Phi_{P}^{-1 / 4}$ \\
\hline$\Phi_{P}>\Phi^{+}$ & $\Phi_{P}^{-1 / 8}$ & $\left(\Phi_{P} \Phi^{+}\right)^{-1 / 8}$ \\
\hline
\end{tabular}


b) Dilute $P$ Chains $\left(\Phi_{P}<1\right)$. - Once again there exists three regions : i) $\Phi_{N}<1$; ii) $1<\Phi_{N}<\Phi^{+}$; iii) $\Phi^{+}<\Phi_{N}$.

\section{i) $\Phi_{N}<1$}

The square region (Fig. 2) $\left[\Phi_{N}, \Phi_{P}<1\right]$ is dilute in both chains and thus all chains are swollen as in a good solvent.

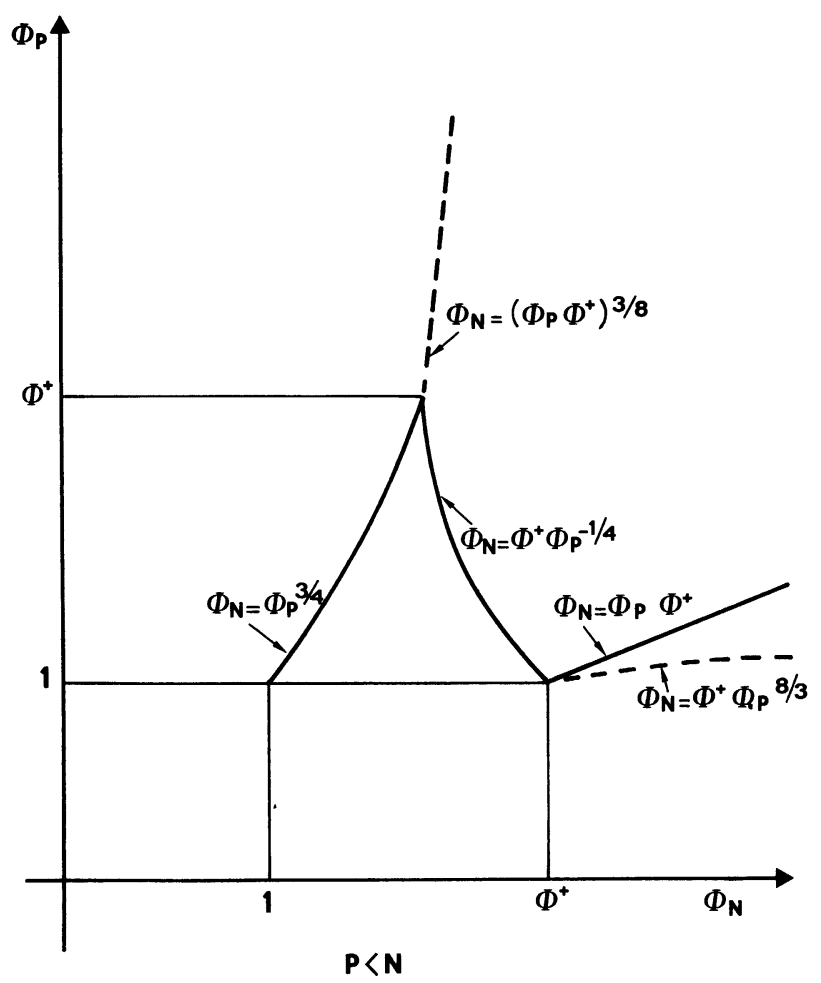

Fig. 2. - The phase diagram described in section 3 .

ii) $1<\Phi_{N}$

For $\Phi_{N}>1$, the $N$ chains overlap and have the usual semi-dilute concentration dependent radius

$$
R_{N}=N^{3 / 5} \Phi_{N}^{-1 / 8},
$$

with a correlation length $\xi_{N}^{0}$ given by (20) with $P \rightarrow 1$. For $\xi_{N}^{0}>R_{P}$, the $P$ chains remain swollen; for $\xi_{N}^{0}<R_{P}$ the swelling forces are screened on a scale $\xi_{N}^{0}$. The condition $\xi_{N}^{0}=R_{P}$ may be expressed

$$
\Phi_{N}=\Phi^{+},
$$

For $\Phi_{N}>\Phi^{+}$, the $P$ chain radii are

$$
R_{P}=P^{1 / 2} \phi_{N}^{-1 / 8} a=P^{3 / 5}\left(\Phi_{N} / \Phi^{+}\right)^{-1 / 8} a .
$$

These results are summarized in table III.

c) $\Phi_{P}>\Phi^{+}$. - We now turn to the situation where $\Phi_{P}>\Phi^{+}$; i.e. dilute $N$ chains are screened with a correlation length $\xi_{P}$. As $\phi_{N}$ increases (for fixed $\phi_{P}$ ), the $N$ chains overlap at a concentration $\left(\Phi_{P} \Phi^{+}\right)^{3 / 8}$ which is the upper dashed line in figure 2 . There is then additional screening by the $N$ chains which becomes dominant when $\xi_{P}=\xi_{N}^{0}$ which is
Table III. - Conformations for $\Phi_{P}<1$.

\begin{tabular}{|l|l|l|}
\hline \multicolumn{1}{|c|}{$\Phi_{N}$} & \multicolumn{1}{|c|}{$R_{\mathrm{P}} / R_{\mathrm{F}}^{P}$} & $R_{N} / R_{\mathrm{F}}^{N}$ \\
\hline$\Phi_{N}<1$ & 1 & 1 \\
\hline$\Phi^{+}>\Phi_{N}>1$ & 1 & $\Phi_{N}^{-1 / 8}$ \\
\hline$\Phi_{N}>\Phi^{+}$ & $\left(\Phi_{N} / \Phi^{+}\right)^{-1 / 8}$ & $\Phi_{N}^{-1 / 8}$ \\
\hline
\end{tabular}

equivalent $\phi_{N}=\phi_{P}$ or $\Phi_{N}=\Phi^{+} \Phi_{P}$. At this boundary $\left(\phi_{N}=\phi_{P}\right)$ there is a crossover to the behaviour characteristic of dilute $P$ chains for $\Phi_{N}>\Phi^{+}$. (Last row of table III.)

d) $\Phi_{N}>\Phi^{+}$. - Similarly for $\Phi_{N}>\Phi^{+}$, even dilute $P$ chains are screened by the $N$ chains. Increasing $\phi_{P}$ (for fixed $\phi_{N}$ ), the semi-dilute $P$ chain regime sets on at $\Phi_{P}=\left(\Phi_{N} / \Phi^{+}\right)^{1 / 8}$ but with no crossover until $\xi_{P}=\xi_{N}^{0}$ as described in $c$ ).

e) $\Phi^{+}>\Phi_{P}>1$. - We finally consider the range of $P$ chain concentrations which act as a moderate solvent for the $N$ chains. As the density, $\Phi_{N}$, of $N$ chain increases for fixed $\Phi_{P}$, the $N$ chains mutually overlap at a concentration $\Phi_{N}=N\left(a / R_{N}\right)^{3}$ which using (28) yields $\Phi_{N}=\Phi_{P}^{3 / 4}$. Above this concentration, the swelling is screened by the interpenetrating $N$ chains. In terms of the renormalized beads (27), the screening length $\xi_{N}$ becomes $(17,20)$.

$$
\xi_{N}=\Phi_{N}^{-3 / 4} \Phi_{P}^{5 / 16} a .
$$

This leads to an $N$ chain radius

$$
R_{N}=\left(N / g_{N}\right)^{1 / 2} \xi_{N}=N^{3 / 5}\left(\Phi_{N} \Phi_{P}^{5 / 4}\right)^{-1 / 8}
$$

which has a proper crossover at $\Phi_{N}=\Phi_{P}^{3 / 4}$ to the dilute result. So long as $\phi_{P}>\phi_{N}$, we know that there exists a quasi-ideal regime for the $N$ chains where

$$
R_{N}=N^{1 / 2} \phi_{P}^{-1 / 8}=N^{3 / 5}\left(\Phi_{P} \Phi^{+}\right)^{-1 / 8} .
$$

This crossover is obtained for

$$
\Phi_{N}=\Phi^{+} \Phi_{P}^{-1 / 4} .
$$

These results are summarized in table IV.

Table IV. - Conformations for $\Phi^{+}>\Phi_{P}>1$.

\begin{tabular}{|l|l|l|}
\hline \multicolumn{1}{|c|}{$\Phi_{N}$} & \multicolumn{1}{c|}{$R_{P} / R_{\mathrm{F}}^{P}$} & \multicolumn{1}{c|}{$R_{N} / R_{\mathrm{F}}^{N}$} \\
\hline$\Phi_{N}<\Phi_{P}^{3 / 4}$ & $\Phi_{P}^{-1 / 8}$ & $\Phi_{P}^{-1 / 4}$ \\
\hline$\Phi_{P}^{3 / 4}<\Phi_{N}<\Phi^{+} \Phi_{P}^{-1 / 4}$ & $\Phi_{P}^{-1 / 8}$ & $\left(\Phi_{N} \Phi_{P}^{5 / 4}\right)^{-1 / 8}$ \\
\hline$\Phi_{P}^{-1 / 4} \Phi^{+}<\Phi_{N}<\Phi_{P} \Phi^{+}$ & $\Phi_{P}^{-1 / 8}$ & $\left(\Phi_{P} \Phi^{+}\right)^{1 / 8}$ \\
\hline$\Phi_{P} \Phi^{+}<\Phi_{N}$ & $\left(\Phi_{N} / \Phi^{+}\right)^{-1 / 8}$ & $\Phi_{N}^{-1 / 8}$ \\
\hline
\end{tabular}


One should notice that in the limit of a melt of $P$ chains with $N^{1 / 2} / P \gg 1\left(\phi_{P}=1\right)$ the results of table IV reduce to those of table I.

4. Summary. - Starting from the Flory result that a long chain $(D P=N)$ in a melt of shorter chains $(D P=P)$ is swollen if $P>N^{1 / 2}$, we have employed scaling arguments and blob concepts to construct the asymptotic dependences of chain conformations for a solution of arbitrary densities of $N$ and $P$ chains in an athermal solvent. The principle regimes are depected in figure 2 and the corresponding conformations in tables II-IV. Neutron scattering experiments on labelled chains [5] may be applied to investigate the phase diagram. This analysis might be considered as the ideal limit of a compatible polymer blend.

Acknowledgments. - We are pleased to acknowledge discussion with F. Brochard, S. Alexander and P. G. De Gennes. This study was partially supported by the US National Science Foundation.

Appendix : Affective medium theory for the excluded volume parameter in a solution of short chains plus athermal solvent. - We consider one long chain in a solution of short chains in an athermal solvent. Outside the long chain coil of radius $R_{N}$, the short chains and the solvent have concentrations $\phi_{P}$ and $\phi_{S}$ with $\phi_{P}+\phi_{S}=1$. Inside the long chain coil these concentrations are modified slightly to $\phi_{P}^{\text {in }}$ and $\phi_{S}^{\text {in }}$ due to the presence of the long chain, with $\phi_{P}^{\text {in }}+\phi_{S}^{\text {in }}$

$$
\phi_{P}^{\text {in }}+\phi_{S}^{\text {in }}+\phi_{N}^{\text {in }}=1 .
$$

Outside the long chain coil, the free energy of mixing is :

$$
f \equiv \frac{F}{T} \sim\left(R_{N} / a\right)^{3}\left[\frac{\phi_{P}}{P} \ln \phi_{P}+\phi_{S} \ln \phi_{S}\right] .
$$

Inside the long chain coil, the free energy of mixing is :

$$
\begin{aligned}
f^{\text {in }} \equiv & \frac{F^{\text {in }}}{T} \sim\left(R_{N} / a\right)^{3} \times \\
& \times\left[\frac{\phi_{P}^{\text {in }}}{P} \ln \phi_{P}^{\text {in }}+\phi_{S}^{\text {in }} \ln \phi_{S}^{\text {in }}+\frac{\phi_{N}^{\text {in }}}{N} \ln \phi_{N}^{\text {in }}\right] .
\end{aligned}
$$

Since the small chains are in equilibrium, we equate their chemical potentials outside $\mu_{P}=\partial f / \partial \phi_{P}$ and inside $\mu_{P}^{\text {in }}=\partial f^{\text {in }} / \partial \phi_{P}^{\text {in }}$, yielding :

$$
\begin{aligned}
\frac{1}{P} \ln \phi_{P}^{\text {in }}-\ln \left(1-\phi_{P}^{\text {in }}-\phi_{N}^{\text {in }}\right) & = \\
= & \frac{1}{P} \ln \phi_{P}-\ln \left(1-\phi_{P}\right) .
\end{aligned}
$$

An identical relation is obtained by equating the chemical potentials for the solvent molecules inside and outside the long chain coil. Since $\phi_{N} \ll 1$, and assuming $\phi_{P}^{\text {in }}=\phi_{P}+\delta \phi_{P}$, we may expand (A.3) to obtain :

$$
\phi_{P}^{\text {in }}=\phi_{P} \frac{1+P \phi_{P}-P \phi_{N}}{1+P \phi_{P}} .
$$

We now modify Edwards' path integral argument [11] to compute the excluded volume parameter for the long chains. The entropic potential associated with the long chain is :

$$
\Phi_{\text {ent }}(r)=-\frac{3 T}{2 a^{2}}\left[\frac{\mathrm{d} R}{\mathrm{~d} n}\right]^{2}
$$

where $a$ is the step length and where $\mathrm{d} r$ is the continuum distance traversed by a continuum number $d n$ of monomers. The excluded volume interaction has a potential proportional to the total concentration of monomers :

$$
\Phi_{\mathrm{ev}}(r) \sim T u \rho_{\text {total }}(r)=T u\left[\rho_{N}(r)+\rho_{P}(r)\right]
$$

where the excluded volume $u \sim a^{3}$. Conservation of «energy» :

$$
\frac{-3 T}{2 a^{2}}\left[\frac{\mathrm{d} r}{\mathrm{~d} n}\right]^{2}+T u\left[\rho_{N}(r)+\rho_{P}(r)\right]=\text { constant }
$$

permits an evaluation of the constant, since at large distances (i.e. outside the coil) $\rho_{N}(r) \rightarrow 0, \rho_{P}(r) \rightarrow \rho_{P}$, $\mathrm{d} r / \mathrm{d} n \rightarrow 0$, whence

$$
\left[\frac{\mathrm{d} r}{\mathrm{~d} n}\right]^{2} \sim a^{2} u\left[\rho_{N}(r)+\rho_{P}(r)-\rho_{P}\right] .
$$

Substituting (A.4), this becomes

$$
\left[\frac{\mathrm{d} r}{\mathrm{~d} n}\right]^{2} \sim a^{2} \tilde{u} \rho_{N}(r)
$$

where

$$
\tilde{u} \equiv \frac{u}{1+P \phi_{P}} .
$$

is the required effective medium coupling constant. 


\section{References}

[1] De Gennes, P. G., Scaling Concepts in Polymer Physics (Cornell U. Press, Ithaca and London) 1979.

[2] Flory, P., J. Chem. Phys. 17 (1949) 303.

[3] We systematically ignore numerical factors and focus on exponents.

[4] The chemical potentials $\mu_{P}^{\text {in }}$ and $\mu_{P}^{\text {out }}$ are derived from the free energies inside and outside the sphere of the long chain respectively :

$$
\begin{aligned}
& F^{\text {in }} / V T=N^{-4 / 3}\left(1-\phi_{P}^{\text {in }}\right)^{1 / 3}+N^{-1}\left(1-\phi_{P}^{\text {in }}\right) \times \\
& \quad \times \ln \left(1-\phi_{P}^{\text {in }}\right)+P^{-1} \phi_{P}^{\text {in }} \ln \phi_{P}^{\text {in }} \\
& F^{\text {out } / V T=}=P^{-1} \phi_{P}^{\text {out }} \ln \phi_{P}^{\text {out }} .
\end{aligned}
$$

[5] Kirste, R. and Lehnen, B., Makromol. Chem. 177 (1976) 1137.

[6] Farnoux, B., Bouk, F., Cotton, J. P., Daoud, M., JanNINCK, G., NiERLiCh, M. and DE GenNes, P. G., J. Physique 39 (1978) 77.

[7] Edwards, S. F., Proc. Phys. Soc. 88 (1966) 265.

[8] Daoud, M., Cotton, J. P., Farnoux, B., Janninck, G., Sarma, G., Benoit, H., Duplessix, R., Picot, C. and DE GenNes, P. G., Macromolecules 8 (1975) 804.

[9] De Gennes, P. G., J. Polym. Sci., Polym. Symp. 61 (1977) 313.

[10] Brochard, F., J. Physique 42 (1981) 505 